\title{
On the computational complexity of read once resolution decidability in $2 \mathrm{CNF}$ formulas
}

\author{
Hans Kleine Büning \\ Computer Science Institute, \\ University of Paderborn \\ kbcsl@uni-paderborn.de
}

\author{
Piotr Wojciechowski* \\ LDCSEE \\ West Virginia University \\ pwjociec@mix.wvu.edu
}

\author{
K. Subramani ${ }^{\dagger}$ \\ LDCSEE \\ West Virginia University \\ k.subramani@mail.wvu.edu
}

September 10, 2018

\begin{abstract}
In this paper, we analyze $2 \mathrm{CNF}$ formulas from the perspectives of Read-Once resolution (ROR) refutation schemes. We focus on two types of ROR refutations, viz., variable-once refutation and clause-once refutation. In the former, each variable may be used at most once in the derivation of a refutation, while in the latter, each clause may be used at most once. We show that the problem of checking whether a given 2CNF formula has an ROR refutation under both schemes is NP-complete. This is surprising in light of the fact that there exist polynomial refutation schemes (tree-resolution and DAG-resolution) for $2 \mathrm{CNF}$ formulas. On the positive side, we show that $2 \mathrm{CNF}$ formulas have copy-complexity 2 , which means that any unsatisfiable $2 \mathrm{CNF}$ formula has a refutation in which any clause needs to be used at most twice.
\end{abstract}

\section{Introduction}

Resolution is a refutation procedure that was introduced in [15] to establish the unsatisfiability of clausal boolean formulas. Resolution is a sound and complete procedure, although it is not efficient in general [14]. Resolution is one among many proof systems (refutation systems) that have been discussed in the literature [18]; indeed it is among the weaker proof systems [11] in that there exist propositional formulas for which short proofs exist (in powerful proof systems) but resolution proofs of unsatisfiability are exponentially long. Resolution remains an attractive option for studying the complexity of constraint classes on account of its simplicity and wide applicability; it is important to note that resolution is the backbone of a range of automated theorem provers [10].

Resolution refutation techniques often arise in proof complexity. Research in proof complexity is primarily concerned with the establishment of non-trivial lower bounds on the proof lengths of propositional tautologies (alternatively refutation lengths of propositional contradictions). An essential aspect of establishing a lower bound is the proof system used to establish the bound. For instance, super-polynomial bounds for tautologies have been established for weak proof systems such as resolution [14]. Establishing that there exist short refutations for all contradictions in a given proof system causes the classes NP and coNP to coincide [13].

There are a number of different types of resolution refutation that have been discussed in the literature [16]. The most important types of resolution refutation are tree-like, dag-like and read-once. Each type of resolution is characterized by a restriction on input clause combination. One of the simplest types of resolution is Read-once Resolution (ROR). In an ROR refutation, each input clause and each derived clause may be used at most once. There are several reasons to prefer a ROR proof over a generalized resolution proof, not the least of which is that ROR proofs must necessarily be of length polynomial (actually, linear) in the size of the input. It follows that ROR cannot be a complete proof system unless NP = coNP. That does not preclude the possibility that we could check in polynomial time whether or not a given CNF formula

\footnotetext{
*This research was supported in part by the National Science Foundation through Award CCF-1305054.

$\dagger$ This work was supported by the Air Force Research Laboratory under US Air Force contract FA8750-16-3-6003. The views expressed are those of the authors and do not reflect the official policy or position of the Department of Defense or the U.S. Government.
} 
has a ROR refutation. Iwama [5] showed that even in case of 3CNF formulas, the problem of checking ROR existence (henceforth, ROR decidability) is NP-complete.

It is well-known that $2 \mathrm{CNF}$ satisfiability is decidable in polynomial time. There are several algorithms for $2 \mathrm{CNF}$ satisfiability, most of which convert the clausal formula into a directed graph and then exploit the connection between the existence of labeled paths in the digraph and the satisfiability of the input formula. A natural progression of this research is to establish the ROR complexity of $2 \mathrm{CNF}$ formulas. We show that the problem of deciding whether an arbitrary $2 \mathrm{CNF}$ formula has a read-once refutation is NP-complete. Although ROR is an incomplete refutation technique, we may be able to find a refutation if clauses can be copied. We show that every $2 \mathrm{CNF}$ formula has an ROR refutation, if every clause can be copied once.

The principal contributions of this paper are as follows:

1. Establishing the computational complexity of ROR decidability for $2 \mathrm{CNF}$ formulas.

2. Establishing the clause copy complexity for ROR existence in $2 \mathrm{CNF}$ formulas.

The rest of this paper is organized as follows: In Section 2, we discuss problem preliminaries and formally define the various types of refutations discussed in this paper. The minimal unsatisfiable subset problems is detailed in Section 3. In Section 4, the Variable Read Once Resolution (VAR-ROR) refutation problem is detailed. We also establish the computational complexity of this problem in $2 \mathrm{CNF}$. We show that ROR decidability for $2 \mathrm{CNF}$ formulas is NP-complete in Section 5. The copy complexity of $2 \mathrm{CNF}$ formulas is established in Section 6. Finally, we conclude in Section 7, by summarizing our contributions and outlining avenues for future research.

\section{Preliminaries}

In this section, we briefly discuss the terms used in this paper. We assume that the reader is familiar with elementary propositional logic. A literal is a variable $x$ or its complement $\neg x . x$ is termed a positive and $\neg x$ is termed a negative literal. A clause is a disjunction of literals. The empty clause, which is always false, is denoted as $\sqcup$.

A Boolean formula $\Phi$ is in CNF, if the formula is a conjunction of clauses. Please note, that a CNF is a set of clauses and written as $\left\{\alpha_{1}, \ldots, \alpha_{n}\right\}, \alpha_{1} \wedge \ldots \wedge \alpha_{n}$, or simply as $\Phi=\alpha_{1}, \ldots, \alpha_{n}$ for clauses $\alpha_{i}$. A formula in CNF is in $k$-CNF, if it is of the form $\alpha_{1} \wedge \alpha_{2} \wedge \ldots \wedge \alpha_{m}$, where each $\alpha_{i}$ is a clause of at most $k$ literals.

For a single resolution step with parent clauses $(\alpha \vee x)$ and $(\neg x \vee \beta)$ with resolvent $(\alpha \vee \beta)$, we write

$$
(\alpha \vee x),(\neg x \vee \beta) \frac{1}{R E S}(\alpha \vee \beta) .
$$

The variable $x$ is termed matching or resolution variable. If for initial clauses $\alpha_{1}, \ldots, \alpha_{n}$, a clause $\pi$ can be generated by a sequence of resolution steps we write

$$
\alpha_{1}, \ldots,\left.\alpha_{n}\right|_{\text {RES }} \pi \text {. }
$$

We now formally define the types of resolution refutation discussed in this paper.

Definition 2.1 A formula is in Var-ROR (variable-read once resolution), if and only if there is a resolution refutation for which every variable is used at most once as a matching variable.

A resolution derivation $\Phi \vdash_{R E S} \pi$ is a Var-ROR derivation, if the matching variables are used at most once. We denote this as $\left.\Phi\right|_{\text {Var-RO-Res }} \pi$.

Definition 2.2 A formula $\Phi$ is said to be minimally Var-ROR, if and only if $\Phi \in$ Var-ROR and every proper sub-formula is not in Var-ROR.

Definition 2.3 A Read-Once resolution refutation is a refutation in which each clause, $\pi$, can be used in only one resolution step. This applies to clauses present in the original formula and those derived as a result of previous resolution steps. 
A resolution derivation $\left.\Phi\right|_{R E S} \pi$ is a read-once resolution derivation, if for all resolution steps $\pi_{1} \wedge \pi_{2} \frac{1}{R E S} \pi$, we delete the clauses $\pi_{1}$ and $\pi_{2}$ from, and add the resolvent $\pi$ to, the current set of clauses. In other words, if $U$ is the current set of clauses, we obtain $U=\left(U \backslash\left\{\pi_{1}, . \pi_{2}\right\}\right) \cup\{\pi\}$. An example of this refutation method can be found in Appendix A.

ROR is the set of formulas in CNF, for which a read-once resolution refutation exists ( $\Phi \in$ ROR if and only if $\left.\left.\Phi\right|_{\text {RO-Res }} \sqcup\right)$.

Example (1): Consider the following 2CNF formula:

$$
\begin{array}{rrr}
\left(x_{1}, x_{2}\right) & \left(x_{3}, x_{4}\right) & \left(\neg x_{1}, \neg x_{3}\right) \\
\left(\neg x_{1}, \neg x_{4}\right) & \left(\neg x_{2}, \neg x_{3}\right) & \left(\neg x_{2}, \neg x_{4}\right)
\end{array}
$$

We now show that this formula does not have a read-once refutation.

To derive $\left(x_{1}\right)$ we need to derive $\left(\neg x_{2}\right)$. Similarly, to derive $\left(x_{2}\right)$ we need to derive $\left(x_{1}\right)$. However, the derivations of both $\left(\neg x_{1}\right)$ and $\left(\neg x_{2}\right)$ require the use of the clause $\left(x_{3}, x_{4}\right)$.

To derive $\left(x_{3}\right)$ we need to derive $\left(\neg x_{4}\right)$. Similarly, to derive $\left(x_{4}\right)$ we need to derive $\left(\neg x_{3}\right)$. However, the derivations of both $\left(\neg x_{3}\right)$ and $\left(\neg x_{4}\right)$ require the use of the clause $\left(x_{1}, x_{2}\right)$.

Definition 2.4 A formula, $\Phi$, is minimally ROR if and only if the formula is in ROR and every proper sub-formula is not in ROR.

It is important to note that both types of Read-Once resolution (Var-ROR and ROR) are incomplete refutation procedures. Furthermore, ROR is a strictly more powerful refutation procedure than VAR-ROR. For instance, consider the formula:

$$
\alpha=\{(a \vee b),(a \vee \neg b),(\neg a \vee b),(\neg a \vee \neg b)\}
$$

It is not hard to see that $\alpha$ has a ROR refutation but not Var-ROR refutation.

After a resolution step $\left(\pi_{1} \vee x\right) \wedge\left(\neg x \vee \pi_{2}\right) \frac{1}{R E S}\left(\pi_{1} \vee \pi_{2}\right)$ in a Var-ROR refutation no clause with a literal over $x$ can be used. Thus, we can delete all clauses which include the variable $x$. We see that Var-ROR $\subseteq$ ROR. For 2-CNF formulas, Var-ROR is a proper subset of ROR.

Example (2): Let $\alpha=\{(a \vee b),(a \vee \neg b),(\neg a \vee b),(\neg a \vee \neg b)\}$. We have that $\alpha \in$ ROR, as shown by the following series of refutation steps:

1. $(a \vee b) \wedge(\neg a \vee b) \frac{1}{R E S}(b)$.

2. $(a \vee \neg b) \wedge(\neg a \vee \neg b) \mid \frac{1}{R E S} \neg(b)$.

3. $(b) \wedge(\neg b) \frac{1}{R E S} \sqcup$.

However, $\alpha$ is not in Var-ROR. After the first resolution step, $(a \vee b) \wedge(\neg a \vee b) \mid \frac{1}{R E S}(b)$, the variable $a$ can not be used as the matching variable in any other resolution step. Thus, we cannot derive $(\neg b)$. Hence there is no Var-ROR refutation.

\section{Minimal Unsatisfiability}

In the characterization of various read-once classes and in the proofs, we make use of minimal unsatisfiable formulas and splitting of these formulas.

First, we recall some notions and results.

Definition 3.1 A formula in CNF is minimal unsatisfiable, if and only if the formula is unsatisfiable and every proper sub-formula is satisfiable. The set of minimal unsatisfiable formulas is denoted as $M U$.

Definition 3.2 The deficiency of a formula $\Phi$, written as $d(\Phi)$, is the number of clauses minus the number of variables. For fixed $k, M U(k)$ is the set of $M U$-formulas with deficiency $k$. 
The problem of deciding whether a formula is minimal unsatisfiable is $\mathbf{D}^{\mathbf{P}}$-complete [8]. $\mathbf{D}^{\mathbf{P}}$ is the class of problems which can be represented as the difference of two NP-problems. Every minimal unsatisfiable formula has a deficiency greater or equal than $1[1]$.

For fixed $k$, deciding if $\Phi \in \mathrm{MU}(k)$ can be solved in polynomial time [3]. For formulas in 2CNF, there is no constant upper bound for the deficiency of minimal unsatisfiable formulas.

The proofs in this paper make use of so-called splitting formulas for MU-formulas.

Definition 3.3 Let

$$
\begin{aligned}
\Phi= & \left(x \vee \pi_{1}\right), \ldots,\left(x \vee \pi_{r}\right), \sigma_{1}, \ldots, \sigma_{t}, \\
& \left(\neg x \vee \phi_{1}\right), \ldots,\left(\neg x \vee \phi_{q}\right)
\end{aligned}
$$

be a minimal unsatisfiable formula, where neither the literal $x$ nor the literal $\neg x$ occur in the clauses $\sigma_{i}$. A pair offormulas $\left(F_{x}, F_{\neg x}\right)$ with $F_{x}=\pi_{1}, \ldots, \pi_{r}, \sigma_{i_{1}}, \ldots, \sigma_{i_{s}}$ and $F_{\neg x}=\sigma_{j_{1}}, \ldots, \sigma_{j_{k}}, \phi_{1}, \ldots, \phi_{q}$ is called a splitting of $\Phi$ over $x$, if $F_{x}$ and $F_{\neg x}$ are minimal unsatisfiable.

Definition 3.4 A splitting $\left(F_{x}, F_{\neg x}\right)$ is disjunctive, if $\left\{\sigma_{i_{1}}, \ldots, \sigma_{i_{s}}\right\} \cap\left\{\sigma_{j_{1}}, \ldots, \sigma_{j_{k}}\right\}$ is empty. That is, $F_{x}$ and $F_{\neg x}$ have no clause $\sigma_{i}$ in common. If additionally $F_{x}$ and $F_{\neg x}$ do not share any variables, then we say that the splitting is variabledisjunctive.

We can continue to split both $F_{x}$ and $F_{\neg x}$ to obtain a splitting tree. Splitting stops when the formula contains only one variable.

Definition 3.5 A splitting tree is complete if every leaf of the tree is a formula that contains at most one variable.

Note that, after splitting on the variable $x$, neither $F_{x}$ nor $F_{\neg x}$ contains the variable $x$. Thus, the splitting tree can have depth at most $(n-1)$.

In case of disjunctive splittings, we speak about disjunctive splitting trees. It is known that formulas in MU(1) have variable-disjunctive splitting trees [2]. Moreover, every minimal unsatisfiable formula with a read-once resolution refutation has a disjunctive splitting tree and vice versa. [6].

Let $\Phi$ be a minimal unsatisfiable 2CNF formula. We will now prove several properties of $\Phi$.

Lemma 3.1 If $\Phi$ contains a unit clause, then $\Phi \in M U(1)$.

Proof: (By induction on the number $n$ of variables) If $\Phi$ has only one variable, then $\Phi=(x) \wedge(\neg x)$. Clearly, $\Phi \in$ MU(1).

Now assume that $\Phi$ has $(n+1)$ variables. Let $(x) \in \Phi$ be a unit clause. Thus, $\Phi$ has the form

$$
\Phi=(x) \wedge\left(\neg x \vee L_{1}\right) \wedge \ldots \wedge\left(\neg x \vee L_{k}\right) \wedge \sigma
$$

where $x$ and $\neg x$ do not occur in any clause in $\sigma$. Thus, the formula

$$
\Phi_{x}=\left(L_{1}\right) \wedge \ldots \wedge\left(L_{k}\right) \wedge \sigma
$$

is minimal unsatisfiable. We have that $\left(L_{1}\right), \ldots,\left(L_{k}\right)$ are unit clauses. Thus, by the induction hypothesis $\Phi_{x} \in \mathrm{MU}(1)$. This means that $\Phi \in \mathrm{MU}(1)$, since $\Phi$ contains one more clause and one more variable than $\Phi_{x}$.

Lemma 3.2 For every variable $x$, the splitting formulas $F_{x}, F_{\neg x}$ are in $M U(1)$.

Proof: We have that the splitting formulas $F_{x}$ and $F_{\neg x}$ contain a unit clause and are minimal unsatisfiable. Thus, from lemma 3.1, $F_{x}, F_{\neg x}$ are in $\mathrm{MU}(1)$.

Lemma 3.3 [7] If for a variable $x$, there is a disjunctive splitting, then the splitting is unique.

Lemma 3.4 The problem of determining if $\Phi$ has a complete disjunctive splitting is in $\mathbf{P}$. Furthermore, if $\Phi$ has a disjunctive splitting for some variable $x$, then there exists a complete splitting tree. 
Proof: Let $\Phi=\left(x \vee L_{1}\right) \wedge\left(x \vee L_{k}\right) \wedge \sigma \wedge\left(\neg x \vee K_{1}\right) \wedge\left(\neg x \vee K_{r}\right)$. For $x$, we take the formula $\left(L_{1}\right) \wedge\left(L_{k}\right) \wedge \sigma$ and reduce the formula to a minimal unsatisfiable formula $F_{x}=\left(L_{1}\right) \wedge\left(L_{k}\right) \wedge \sigma_{1}$ where $\sigma_{1} \subseteq \sigma$. This can be done in polynomial time, because the satisfiability problem for $2-\mathrm{CNF}$ is solvable in linear time. Similarly, for $\neg x$, we can compute $F_{\neg x}$.

To decide whether the formula has a disjunctive splitting tree, it suffices to look for a variable $x$ for which $\left(F_{x}, F_{\neg x}\right)$ is a disjunctive splitting. From lemma 3.2, $F_{x}$ and $F_{\neg x}$ are in MU(1) and therefore have disjunctive splitting trees [2].

For each $x$, we compute a splitting $\left(F_{x}, F_{\neg x}\right)$. If this splitting is disjunctive, we have found the desired splitting. If it is not disjunctive, then for $x$ there is no disjunctive splitting. If $x$ did have a disjunctive splitting, then, by lemma 3.3 , that splitting would be unique meaning that $x$ would have no non-disjunctive splittings.

Hence, the problem is solvable in polynomial time.

\section{The Complexity of Var-ROR for 2CNF}

In this section, we show that determining if a formula in 2CNF has a variable read-once refutation is NP-complete. The Var-ROR problem is NP-complete for CNF formulas in general [17]. We show that this result holds even when restricted to $2 \mathrm{CNF}$ formulas.

Let $\Phi$ be a formula in 2CNF.

Theorem $4.1 \Phi \in$ Var-ROR, if and only if there exists a sub-formula $\Phi^{\prime} \subseteq \Phi$ such that $\Phi^{\prime} \in M U(1)$.

Theorem 4.1 follows immediately from lemma 4.1.

Lemma 4.1 $\Phi$ is minimally Var-ROR, if and only if $\Phi \in M U(1)$.

Proof: Let $\Phi$ be minimally Var-ROR. We will show that $\Phi \in \operatorname{MU}(1)$ by induction on the number of variables. First, assume that $\Phi$ is a CNF formula over 1 variable. Thus, $\Phi$ has the form $(x) \wedge(\neg x)$. Obviously, $\Phi \in$ MU(1). Now assume that $\Phi$ is a CNF formula over $(n+1)$ variables. Let

$$
(\alpha \vee x),(\neg x \vee \beta) \frac{1}{R E S}(\alpha \vee \beta)
$$

be the first resolution step in a Var-ROR refutation of $\Phi$. Thus, the formula

$$
\Phi^{\prime}=(\Phi \backslash\{(\alpha \vee x),(\neg x \vee \beta)\} \cup\{(\alpha \vee \beta)\})
$$

is minimally Var-ROR and contains no clause with $x$ or $\neg x . x$ has already been used as a matching variable. Thus, if any other clauses of $\Phi$, or $\Phi^{\prime}$, used $x$ then $\Phi$ would not be minimally Var-ROR.

$\Phi^{\prime}$ has $n$ variables. Thus, by the induction hypothesis $\Phi^{\prime} \in \operatorname{MU}(1)$. Since $\Phi^{\prime}$ is minimal unsatisfiable and consists of $(n+1)$ clauses, $\Phi$ is minimal unsatisfiable and consists of $(n+2)$ clauses. This means that $\Phi \in \operatorname{MU}(1)$.

Now let $\Phi$ be a formula in MU(1). We will show that $\Phi$ is minimally Var-ROR by induction on the number of variables.

For every formula in MU(1), there exists a variable-disjunctive splitting tree [2]. Thus, we can easily construct a Var-ROR refutation for $\Phi$.

First, assume that $\Phi$ is a CNF formula over 1 variable. Thus, $\Phi=(x) \wedge(\neg x)$ and is minimally Var-ROR.

Now assume that $\Phi$ is a CNF formula over $(n+1)$ variables. Let $\left(F_{x}, F_{\neg x}\right)$ be the first variable-disjunctive splitting in a variable-disjunctive splitting tree. Without loss of generality we assume that neither $F_{x}$ nor $F_{\neg x}$ is the empty clause. By the induction hypothesis, both formulas are minimally Var-ROR, because, by lemma $3.2, F_{x}, F_{\neg x} \in \mathrm{MU}(1)$.

Thus, there is a Var-ROR derivation $\left.F_{x}^{x}\right|_{\text {Var-RO-Res }}(x)$ and $\left.F_{\neg x}^{\neg x}\right|_{\text {Var-RO-Res }}(\neg x)$, where $F_{x}^{x}\left(F_{\neg x}^{\neg x}\right)$ is the formula we obtain by adding the removed literal $x(\neg x)$ to the clauses in $F_{x}\left(F_{\neg x}\right)$. The final step is to resolve $(x)$ and $(\neg x)$. Note that the variable $x$ has not been used as a matching variable in the derivations $\left.F_{x}^{x}\right|_{\text {Var-RO-Res }}(x)$ and $\left.F_{\neg x}^{\neg x}\right|_{\text {Var-RO-Res }}(\neg x)$. Hence, $\Phi$ is in Var-ROR and is minimally Var-ROR.

For a formula in MU(1), there always exists a complete variable-disjunctive splitting. Furthermore, a complete variabledisjunctive splitting tree can be computed in polynomial time. Thus, every formula in MU(1) has a Var-ROR refutation that can be computed in polynomial time.

Corollary 4.1 Every formula in MU(1) has a Var-ROR refutation that can be computed in polynomial time. 
Next, we will show that determining if a 2CNF formula has a Var-ROR refutation is NP-complete. It can easily be seen that this problem is in NP. If the formula has $n$ variables, then at most $n$ resolution steps can be performed. The NP-hardness will be shown by a reduction from the vertex-disjoint path problem for directed graphs.

Definition 4.1 Given a directed graph $G$ and pairwise distinct vertexes $s_{1}, t_{1}, s_{2}, t_{2}$, the vertex-disjoint path problem $(2-D P P)$ consists of finding a pair of vertex-disjoint paths in $G$, one from $s_{1}$ to $t_{1}$ and the other from $s_{2}$ to $t_{2}$.

The problem is known to be NP-complete [4]. Now we modify the problem as follows.

Definition 4.2 Given a directed graph $G$ and two distinct vertexes $s$ and $t$, the vertex-disjoint cycle problem $(C$-DPP) consists of finding a pair of vertex-disjoint paths in $G$, one from s to $t$ and the other from $t$ to $s$.

Note that the paths are vertex-disjoint, if the inner vertexes of the path from $s$ to $t$ are disjoint from the inner vertexes of the path from $t$ to $s$.

\section{Lemma 4.2 C-DPP is NP-complete.}

Proof: Obviously, the problem is in NP. We will show NP-hardness by a reduction from 2-DPP.

From $G=(V, E), s_{1}, t_{1}, s_{2}$, and $t_{2}$ we construct the new graph

$$
G^{\prime}=\left(V \cup\{s, t\}, E \cup\left\{\left(s, s_{1}\right),\left(t_{2}, s\right),\left(t_{1}, t\right),\left(t, s_{2}\right)\right\}\right) .
$$

Assume that $G$ has two vertex-disjoint paths, $w_{1}$ from $s_{1}$ to $t_{1}$, and $w_{2}$ from $s_{2}$ to $t_{2}$. Thus, the paths $\left(s, s_{1}\right), w_{1},\left(t_{1}, t\right)$ and $\left(t, s_{2}\right), w_{2},\left(t_{2}, s\right)$ in $G^{\prime}$ are vertex-disjoint. Note that $s_{1}, s_{2}, t_{1}, t_{2}$ are pairwise distinct. Thus, $G^{\prime}$ has the desired vertex-disjoint cycle.

Now assume that $G^{\prime}$ has two vertex-disjoint paths, $w_{1}$ from $s$ to $t$, and $w_{2}$ from $t$ to $s$. By construction, $w_{1}$ must contain a path from $s_{1}$ to $t_{1}$. Similarly, $w_{2}$ must contain a path from $s_{2}$ to $t_{2}$. Since $w_{1}$ and $w_{2}$ are vertex-disjoint these new paths must also be vertex-disjoint. Thus, $G$ has the desired vertex-disjoint paths.

Theorem 4.2 Determining if a 2 CNF formula has a Var-ROR refutation is NP-complete.

Proof: As previously stated, we only need to show NP-hardness. That will be done by a reduction from C-DPP. From $G=(V, E), s$, and $t$ we construct a formula $\Phi$ in $2 \mathrm{CNF}$ as follows:

1. For each vertex $v_{i} \in V-\{s, t\}$, create the variable $x_{i}$.

2. Create the variable $x_{0}$.

3. Let $v_{i}, v_{j} \in V-\{s, t\}$.

(a) If $\left(s, v_{i}\right) \in E$ add the clause $\left(x_{0} \rightarrow x_{i}\right)$ to $\Phi$.

(b) If $\left(t, v_{i}\right) \in E$ add the clause $\left(\neg x_{0} \rightarrow x_{i}\right)$ to $\Phi$.

(c) If $\left(v_{i}, s\right) \in E$ add the clause $\left(x_{i} \rightarrow x_{0}\right)$ to $\Phi$.

(d) If $\left(v_{i}, t\right) \in E$ add the clause $\left(x_{i} \rightarrow \neg x_{0}\right)$ to $\Phi$.

(e) If $\left(v_{i}, v_{j}\right) \in E$ add the clause $\left(x_{i} \rightarrow x_{j}\right)$ to $\Phi$.

Assume that $G$ has two vertex-disjoint paths,

$$
w_{1}=s, v_{i_{1}}, \ldots, v_{i_{j}}, t \text { and } w_{2}=t, v_{i_{j+1}}, \ldots, v_{i_{k}}, s .
$$

Thus, there exist $2 \mathrm{CNF}$ formulas $\Phi_{1}$ and $\Phi_{2}$ such that:

$$
\begin{aligned}
& \Phi_{1}=\left\{\left(x_{0} \rightarrow x_{i_{1}}\right),\left(x_{i_{1}} \rightarrow x_{i_{2}}\right), \ldots,\left(x_{i_{j}} \rightarrow \neg x_{0}\right)\right\} \\
& \Phi_{2}=\left\{\left(\neg x_{0} \rightarrow x_{i_{j+1}}\right),\left(x_{i_{j+1}} \rightarrow x_{i_{j+2}}\right), \ldots,\left(x_{i_{k}} \rightarrow x_{0}\right)\right\} .
\end{aligned}
$$


Clearly, $\left.\Phi_{1}\right|_{\text {Var-RO-Res }}\left(\neg x_{0}\right)$ and $\left.\Phi_{2}\right|_{\text {Var-RO-Res }}\left(x_{0}\right)$. Note that $x_{0}$ has not been used as a matching variable. Since $w_{1}$ and $w_{2}$ are vertex-disjoint, we have that

$$
\left\{x_{i_{1}}, \ldots, x_{i_{j}}\right\} \cap\left\{x_{i_{j+1}}, \ldots, x_{i_{k}}\right\}=\emptyset \text {. }
$$

Thus, $\left.\Phi_{1} \cup \Phi_{2}\right|_{\text {Var-RO-Res }} \sqcup$. This means that $\Phi \supseteq \Phi_{1} \cup \Phi_{2}$ is in Var-ROR.

Now assume that $\Phi$ is in Var-ROR. Let $\Phi^{\prime} \subseteq \Phi$ be minimally Var-ROR. We have that $\Phi^{\prime}$ contains clauses with $x_{0}$ and $\neg x_{0}$. Otherwise, the formula would be satisfiable by setting each $x_{i}$ to true.

We proceed by an induction on the number of clauses in $\Phi^{\prime}$.

The shortest formula is $\Phi^{\prime}=\left(x_{0} \rightarrow \neg x_{0}\right) \wedge\left(\neg x_{0} \rightarrow x_{0}\right)$. This $\Phi^{\prime}$ is generated when $(s, t),(t, s) \in E$. These edges form the desired vertex-disjoint paths.

Let $y$ be the variable for which $(y) \wedge(\neg y) \mid \frac{1}{R E S} \sqcup$ is the last resolution step in $\left.\Phi^{\prime}\right|_{\text {Var-RO-Res }} \sqcup$. Thus, $\Phi^{\prime}$, can be divided into two variable-disjoint sets of clauses, $\Phi_{1}^{\prime}$ and $\Phi_{2}^{\prime}$, such that $\left.\Phi_{1}^{\prime}\right|_{\text {Var-RO-Res }}(y)$ and $\left.\Phi_{2}^{\prime}\right|_{\text {Var-RO-Res }}(\neg y)$. Otherwise, a variable would be used twice in $\Phi^{\prime} \bigvee_{\text {Var-RO-Res }} \sqcup$.

Let $\left(L \vee x_{i}\right) \wedge\left(\neg x_{i} \vee K\right) \frac{1}{{ }_{R E S}}(L \vee K)$ a resolution step in $\Phi_{1}^{\prime} \vdash_{\text {Var-RO-Res }}(y)$ such that $\left(L \vee x_{i}\right) \in \Phi_{1}^{\prime}$ and $\left(\neg x_{i} \vee K\right) \in \Phi_{1}^{\prime}$. Thus, no clause with $x_{i}$ occurs in $\Phi_{2}^{\prime}$ or $\Phi_{1}$ (except $\left(L \vee x_{i}\right)$ and $\left(\neg x_{i} \vee K\right)$ ). Moreover, we see that the formula

$$
\left(\Phi^{\prime} \backslash\left\{\left(L \vee a_{i}\right),\left(\neg a_{i} \vee K\right)\right\}\right) \cup\{(L \vee K)\}
$$

is in Var-ROR. This formula represents the reduced graph where the edges $L \rightarrow a_{i}$ and $a_{i} \rightarrow K$ are replaced with the edge $L \rightarrow K$. By the induction hypothesis, there exists a vertex-disjoint cycle in this reduced graph. Thus, a vertex-disjoint cycle exists in $G$.

For arbitrary formulas in CNF, the problem of deciding whether a formula $\Phi$ has a sub-formula $\Phi^{\prime}$ such that $\Phi^{\prime} \in M U(1)$ is known to be NP-complete. But it was only known for arbitrary CNF. Based on the Theorems above, we obtain as a corollary that the MU(1) sub-formula problem is NP-complete for 2CNF, too.

Corollary 4.2 The problem of deciding whether a formula in 2CNF contains a minimal unsatisfiable formula with deficiency 1 is NP-complete.

\section{The Complexity of ROR for $2 \mathrm{CNF}$}

In this section, we show that determining if a formula in $2 \mathrm{CNF}$ has a read-once refutation is NP-complete. It was established in [17] that the Var-ROR problem for $2 \mathrm{CNF}$ can be reduced to the ROR problem for $2 \mathrm{CNF}$. Together with Theorem 4.2 , this establishes that the ROR problem for $2 \mathrm{CNF}$ is NP-complete. We now present an alternate way of obtaining this result.

Unlike minimally Var-ROR formulas, minimally ROR formulas are not necessarily minimal unsatisfiable. They also can have deficiencies other than 1. An example of such a formula can be seen in Appendix B.

We now prove some properties of minimal unsatisfiable formulas in 2-CNF with one or two unit clauses. It can easily be seen that such formulas contain at most two unit clauses.

Lemma 5.1 Let $\Phi$ be a minimal unsatisfiable $2 C N F$ formula.

1. If $\Phi$ contains two unit clauses, then $\Phi$ has the form

$$
(L),\left(\neg L \vee L_{1}\right), \ldots,\left(\neg L_{t-1} \vee L_{t}\right),\left(\neg L_{t} \vee \neg K\right),(K)
$$

where $L, L_{1}, \ldots, L_{t}, K$ are pairwise distinct.

2. If $\Phi$ contains exactly one unit clause, then $\Phi$ has the form

$$
\begin{array}{r}
(L),\left(\neg L \vee L_{1}\right),\left(\neg L_{1} \vee L_{2}\right), \ldots,\left(\neg L_{t} \vee K\right), \\
\left(\neg K \vee S_{1}\right),\left(\neg S_{1} \vee S_{2}\right), \ldots,\left(\neg S_{q} \vee R\right), \\
\left(\neg K \vee P_{1}\right),\left(\neg P_{1} \vee P_{2}\right), \ldots,\left(\neg P_{m} \vee \neg R\right)
\end{array}
$$

where the literals are all pairwise distinct. 
3. If $\Phi$ contains at least one unit clause, then $\Phi$ has a read-once resolution refutation.

4. If $\Phi$ is in $M U(1)$, then $\Phi$ has a ROR refutation.

Proof: We prove each part of the lemma separately.

The proofs of part 1 and 2 are straightforward because no minimal unsatisfiable 2CNF formula contains more than two unit clauses.

3. If the formula has two unit clauses, then structure of the formula leads immediately to a ROR refutation.

If the formula has one unit clause, then we can perform the desired resolution refutation as follows:

(a) First, we resolve $\left(\neg K \vee S_{1}\right),\left(\neg S_{1} \vee S_{2}\right), \ldots,\left(\neg S_{q} \vee R\right)$ to obtain $(\neg K \vee R)$.

(b) Then, we resolve $\left(\neg K \vee P_{1}\right),\left(\neg P_{1} \vee P_{2}\right), \ldots,\left(\neg P_{m} \vee \neg R\right)$ to obtain $(\neg K \vee \neg R)$.

(c) Next, we perform the resolution step

$$
(\neg K \vee R) \wedge(\neg K \vee \neg R) \mid \frac{1}{R E S}(\neg K) .
$$

(d) Finally, the unit clause $(\neg K)$ together with the chain

$$
(L),\left(\neg L \vee L_{1}\right),\left(\neg L_{2} \vee L_{3}\right), \ldots,\left(\neg L_{t} \vee K\right)
$$

resolve to finish the ROR refutation.

4. Every $2 \mathrm{CNF}$ formula in MU(1) has a complete disjunctive splitting tree. This guarantees the existence of a ROR refutation [6].

Theorem 5.1 Let $\Phi$ be in $2 C N F$. $\Phi$ is in ROR, if and only if there exists a sub-formula $\Phi^{\prime} \subseteq \Phi$ for which there exists a variable $x$ and a disjunctive splitting $\left(F_{x}, F_{\neg x}\right)$ over $x$, such that $F_{x}, F_{\neg x}$ are in $M U(1)$.

Proof: Suppose, there exists a sub-formula $\Phi^{\prime} \subseteq \Phi$ with disjunctive splitting $\left(F_{x}, F_{\neg x}\right)$, where $F_{x}, F_{\neg x} \in \mathrm{MU}(1)$. We have that $F_{x}$ and $F_{\neg x}$ each contain at least one unit clause. Now we reconstruct the clauses of $F_{x}$ and $F_{\neg x}$ by adding the removed literal $x$ (resp. $\neg x$ ) to the clauses in $F_{x}\left(\right.$ resp. $F_{\neg x}$ ). These new formulas are denoted as $F_{x}^{x}$ and $F_{\neg x}$.

From lemma 5.1, every formula in $\mathrm{MU}(1)$ with a unit-clause has a read-once resolution refutation. We also have that $(x)$ and $(\neg x)$ do not occur in the splitting formulas. Thus, we get $F_{x} \vdash_{R O-R e s} \sqcup, F_{\neg x} \vdash_{R O-R e s} \sqcup, F_{x}^{x} \vdash_{R O-R e s}(x)$, and $\left.F_{\neg x}\right|_{\text {RO-Res }}(\neg x)$. Now we have to guarantee that there is a read-once resolution for $\Phi$. $\left(F_{x}, F_{\neg x}\right)$ is disjunctive splitting. Thus, no clause of $\Phi$ occurs in both $F_{x}^{x}$ and in $F_{\neg x}^{\neg x}$. We can combine the resolutions $\left.F_{x}^{x}\right|_{R O-R e s}(x)$ and $\left.F_{\neg x}^{\neg x}\right|_{R O-R e s}(\neg x)$, with the resolution step $(x) \wedge(\neg x) \frac{1}{R E S} \sqcup$ to yield $\left.\Phi\right|_{R O \text {-Res }} \sqcup$, since $F_{x}^{x}, F_{\neg x}^{\neg x} \subseteq \Phi$.

Now suppose, $\Phi \in$ ROR and without loss of generality is minimally ROR. We will show that $\Phi$ contains the desired splitting. Let $\left.x \wedge \neg x\right|_{R E S} \sqcup$ the last resolution step in the read-once resolution refutation. Furthermore, let $F_{x}^{x}\left(F_{\neg x}^{\neg x}\right.$ respectively) be the set of original clauses from $\Phi$ used in the derivation of $x$ ( $\neg x$ respectively). These sets have no clause in common because together they form a read-once resolution refutation for $\Phi$.

The formulas have the form

$$
F_{x}^{x}=\left(x \vee L_{1}\right) \wedge \ldots \wedge\left(x \vee L_{t}\right) \wedge \sigma_{1} \text { and } F_{\neg x}^{\neg x}=\left(\neg x \vee K_{1}\right) \wedge \ldots \wedge\left(\neg x \vee K_{r}\right) \wedge \sigma_{2},
$$

where $\sigma_{1} \cap \sigma_{2}=\emptyset$.

Thus, we can construct the formulas

$$
F_{x}=\left(L_{1}\right) \wedge \ldots \wedge\left(L_{t}\right) \wedge \sigma_{1} \text { and } F_{\neg x}=\left(K_{1}\right) \wedge \ldots \wedge\left(K_{r}\right) \wedge \sigma_{2},
$$

where $\sigma_{1} \cap \sigma_{2}=\emptyset$.

By construction, $\left.F_{x}\right|_{R O-R e s} \sqcup$ and $\left.F_{\neg x}\right|_{R O-R e s} \sqcup$. Both $F_{x}$ and $F_{\neg x}$ are minimal unsatisfiable. Otherwise, $\Phi$ would not be minimally ROR. Thus, $\left(F_{x}, F_{\neg x}\right)$ is a disjunctive splitting. Both $F_{x}$ and $F_{\neg x}$ contain unit clauses. Thus, by lemma 3.1, $F_{x}$ and $F_{\neg x}$ are $\mathrm{MU}(1)$.

We will now prove the NP-completeness or the ROR problem for $2 \mathrm{CNF}$ formulas. Instead of using the vertex-disjoint cycle problem, we will be reducing from the edge-disjoint cycle problem for directed graphs. 
Definition 5.1 Given a directed graph $G$ and two distinct vertexes $s$ and $t$, the edge-disjoint cycle problem $(C-D E P)$ consists of finding a pair of edge-disjoint paths in $G$, one from $s$ to $t$ and the other from $t$ to $s$.

The problem is NP-complete. For two pairs of vertexes, the edge-disjoint path problem is NP-complete [9]. We can reduce the edge-disjoint path problem to C-DEP the same way we reduced 2-DPP to C-DPP.

\section{Theorem 5.2 The ROR problem for 2 CNF formulas is NP-complete.}

Proof: ROR is in NP for arbitrary formulas in CNF [5]. Thus, we only need to show NP-hardness. That will be done by a reduction from C-DEP.

From $G=(V, E), s$, and $t$ we construct a formula $\Phi$ in $2 \mathrm{CNF}$ as follows:

1. For each vertex $v_{i} \in V-\{s, t\}$, create the variable $x_{i}$.

2. Create the variable $x_{0}$.

3. Let $v_{i}, v_{j} \in V-\{s, t\}$.

(a) If $\left(s, v_{i}\right) \in E$ add the clause $\left(x_{0} \rightarrow x_{i}\right)$ to $\Phi$.

(b) If $\left(t, v_{i}\right) \in E$ add the clause $\left(\neg x_{0} \rightarrow x_{i}\right)$ to $\Phi$.

(c) If $\left(v_{i}, s\right) \in E$ add the clause $\left(x_{i} \rightarrow x_{0}\right)$ to $\Phi$.

(d) If $\left(v_{i}, t\right) \in E$ add the clause $\left(x_{i} \rightarrow \neg x_{0}\right)$ to $\Phi$.

(e) If $\left(v_{i}, v_{j}\right) \in E$ add the clause $\left(x_{i} \rightarrow x_{j}\right)$ to $\Phi$.

Assume that $G$ has two edge-disjoint paths,

$$
w_{1}=s, v_{i_{1}}, \ldots, v_{i_{j}}, t \text { and } w_{2}=t, v_{i_{j+1}}, \ldots, v_{i_{k}}, s .
$$

Thus, there exist $2 \mathrm{CNF}$ formulas $\Phi_{1}$ and $\Phi_{2}$ such that:

$$
\begin{aligned}
& \Phi_{1}=\left\{\left(x_{0} \rightarrow x_{i_{1}}\right),\left(x_{i_{1}} \rightarrow x_{i_{2}}\right), \ldots,\left(x_{i_{j}} \rightarrow \neg x_{0}\right)\right\} \\
& \Phi_{2}=\left\{\left(\neg x_{0} \rightarrow x_{i_{j+1}}\right),\left(x_{i_{j+1}} \rightarrow x_{i_{j+2}}\right), \ldots,\left(x_{i_{k}} \rightarrow x_{0}\right)\right\} .
\end{aligned}
$$

Obviously, $\Phi_{1} \vdash_{R O-R e s}\left(\neg x_{0}\right)$ and $\Phi_{2} \vdash_{R O-R e s}\left(x_{0}\right)$. Note that $x_{0}$ has not been used as a matching variable. Since $w_{1}$ and $w_{2}$ are edge-disjoint, we have that $\Phi_{1} \cap \Phi_{2}=\emptyset$. Thus, $\left.\Phi_{1} \cup \Phi_{2}\right|_{R O-R e s} \sqcup$. This means that $\Phi \supseteq \Phi_{1} \cup \Phi_{2}$ is in ROR.

Now assume that $\Phi$ is in ROR.

Let $\Phi^{\prime} \subseteq \Phi$ be minimally ROR. We have that $\Phi^{\prime}$ contains clauses with $x_{0}$ and $\neg x_{0}$. Otherwise, the formula would be satisfiable by setting each $x_{i}$ to true.

We proceed by an induction on the number of clauses in $\Phi^{\prime}$.

The shortest formula is $\Phi^{\prime}=\left(x_{0} \rightarrow \neg x_{0}\right) \wedge\left(\neg x_{0} \rightarrow x_{0}\right)$. This $\Phi^{\prime}$ is generated when $(s, t),(t, s) \in E$. These edges form the desired edge-disjoint paths.

Let $(L \rightarrow K) \wedge(K \rightarrow R) \frac{1}{R E S}(L \vee R)$ be a resolution step where $(L \rightarrow K) \in \Phi^{\prime}$ and $(K \rightarrow R) \in \Phi^{\prime}$. Note that $(L \rightarrow R) \notin \Phi^{\prime}$. Otherwise, $\Phi^{\prime}$ would not be minimally ROR.

In a read-once refutation, we remove the parent clauses from $\Phi$ and add the resolvent $(L \rightarrow R)$. This new formula has a read-once resolution refutation and can be considered as obtained by a reduced graph without the edges $L \rightarrow K, K \rightarrow R$ but with the edge $L \rightarrow R$. By the induction hypothesis, this new graph contains the desired edge-disjoint cycle. If we replace the edge $L \rightarrow R$ in this cycle with $L \rightarrow K$ and $K \rightarrow R$, then we construct the desired edge-disjoint cycle in $G$.

By lemma 3.4, the problem of determining if an MU-formula in 2CNF has a disjunctive splitting whose splitting formulas are in $\mathrm{MU}(1)$ can be decided in polynomial time. Hence, for MU-formulas in 2CNF, the ROR problem is solvable in polynomial time.

Corollary 5.1 The ROR problem for minimal unsatisfiable $2 C N F$ formulas is in $\mathbf{P}$. 


\section{Copy Complexity of $2 \mathrm{CNF}$ formulas}

Let $\Phi$ be an unsatisfiable formula in CNF and let $\Lambda$ be a tree-like resolution refutation of $\Phi$. For each clause $\pi_{i} \in \Phi$, let $\Lambda_{i}$ be the number of times $\pi_{i}$ is used in a resolution step of $\Lambda$.

Definition 6.1 A CNF formula $\Phi$ has a Copy Complexity of $k$, if there exists $\Lambda$ such that $\Lambda_{i} \leq k$ for $i=1, \ldots, m$.

Note that in a tree-like refutation reusing a resultant clause requires the reuse of the clauses originally in $\Phi$. Thus, by limiting the number of times $\Lambda$ can use each clause in $\Phi$ we also limit the number of times each resultant clause can be used.

We can equivalently define copy complexity as follows.

Definition 6.2 A CNF formula $\Phi$ has a Copy Complexity of $k$, if there exists a multi-set of CNF clauses, $\Phi^{\prime}$ such that:

1. Every clause in $\Phi$ appears at most $k$ times in $\Phi^{\prime}$.

2. Every clause in $\Phi^{\prime}$ appears in $\Phi$.

3. $\Phi^{\prime}$ has a read-once refutation.

Thus, if a formula, $\Phi$, has a copy complexity of 1 then the formula has a read-once resolution refutation.

We can extend the concept of copy complexity to classes of CNF formulas.

Definition 6.3 A class of CNF formulas has a Copy Complexity of $k$, if every formula in that class has a copy complexity of $k$.

We will now show that $2 \mathrm{CNF}$ has a copy complexity of 2 . This means that we can always prove unsatisfiablity using each clause at most twice.

Lemma 6.1 Let $\Phi$ be a formula in $2 C N F$. If we can prove $\left(x_{i}\right)$, then we can prove $\left(x_{i}\right)$ using each clause no more than once.

Proof: For a formula $\Phi$, we construct the corresponding implication graph $G$ as follows:

1. For each variable $x_{i}$, we create the verticies $x_{i}$ and $\bar{x}_{i}$. These correspond to the literals $x_{i}$ and $\neg x_{i}$.

2. For each clause $(L \vee K)$, we create the edges edges $\bar{L} \rightarrow K$ and $\bar{K} \rightarrow L$.

3. For each unit clause $(L)$, we create the edge $\bar{L} \rightarrow L$.

We know that we can prove $\left(x_{i}\right)$ in $\Phi$, if and only if there exists a path from $\bar{x}_{i}$ to $x_{i}$ in $G$. Let $p$ denote this path. If no two edges in $p$ correspond to the same clause, then this path already corresponds to a read-once proof of $\left(x_{i}\right)$.

Let $e$ be the first edge in $p$ such that the other edge corresponding to the same clause as $e$ has already been used in $p$. We can assume without loss of generality that $e$ is $x_{j} \rightarrow x_{k}$. Thus, we can break $p$ up as follows:

1. a path, $p_{1}$, from $\bar{x}_{i}$ to $\bar{x}_{k}$,

2. the edge $\bar{x}_{k} \rightarrow \bar{x}_{j}$ (the edge corresponding to the same clause as $e$ ),

3. a path, $p_{2}$, from $\bar{x}_{j}$ to $x_{j}$,

4. the edge $x_{j} \rightarrow x_{k}$ (the edge $e$ ),

5. and a path, $p_{3}$, from $x_{k}$ to $x_{i}$.

This can be seen in Figure 1.

By our choice of $e$, we know no two edges in $p_{1}$ or $p_{2}$ correspond to the same clause. Thus, $p_{2}$ corresponds to a a read-once proof of $\left(x_{j}\right)$. We also have that $p_{1}$ combined with the edge $\bar{x}_{k} \rightarrow \bar{x}_{j}$ is a read once proof of $\left(\neg x_{i} \rightarrow \neg x_{j}\right)$. Combining these two yields a read-once proof of $\left(\neg x_{i} \rightarrow x_{i}\right)$ and therefore for $\left(x_{i}\right)$. 


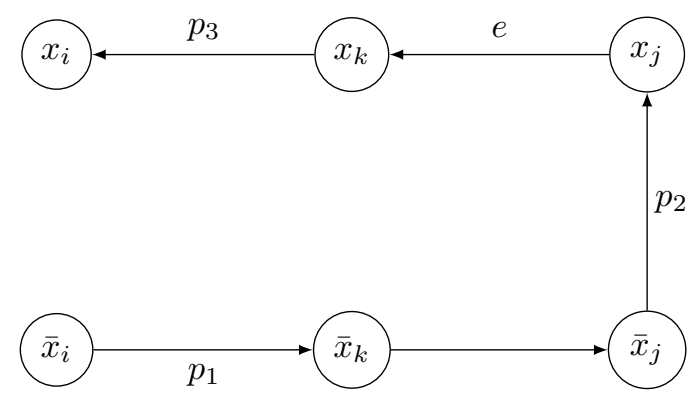

Figure 1: Example of Path $p$

Theorem 6.1 If a formula in $2 C N F$ is unsatisfiable, then there exists a resolution refutation in which no clause is used more than twice.

Proof: Suppose, the formula $\Phi$ in $2 \mathrm{CNF}$ is unsatisfiable. Then for some $x_{i}$, we have that:
1. $\Phi \vdash_{R E S}\left(x_{i}\right)$
2. and $\Phi \vdash_{R E S}\left(\neg x_{i}\right)$.

From Lemma 6.1, we know that we can derive $\left(x_{i}\right)$ using each clause at most once. Similarly we can derive $\left(\neg x_{i}\right)$ using each clause at most once.

Thus, for $\Phi$, there is a resolution refutation using each clause at most twice. That is once in the refutation derivation of $\left(x_{i}\right)$ and once in the resolution derivation of $\left(\neg x_{i}\right)$.

\section{Conclusion}

In this paper, we discuss the computational complexity of ROR decidability in a well-known classe of CNF formulas, viz., 2CNF. Prior research had established that the ROR decidability problem is NP-complete for 3CNF formulas. Likewise, it is known that resolution refutations for $3 \mathrm{CNF}$ formulas, even when they exist need not be polynomial sized. For $2 \mathrm{CNF}$ formulas, it is well-known that polynomial sized refutations exist in the general case. We showed that ROR decidability in $2 \mathrm{CNF}$ formulas is NP-complete. Additionally, we showed that the copy complexity of $2 \mathrm{CNF}$ formulas is 2 . This means that every unsatisfiable $2 \mathrm{CNF}$ formula has a refutation in which each input clause is used at most twice. Furthermore, the optimal length resolution when clause copy is permitted can be determined in polynomial time.

\section{References}

[1] R.Aharoni and N. Linial, Minimal non-two-colorable hypergraphs and minimal unsatisfiable formulas, J. Combin.Theory Ser. A, 43:196-204, 1986.

[2] G. Davidov, I. Davydova, and H. Kleine Büning. An efficient algorithm for the minimal unsatisability problem for a subclass of CNF. Annals of Mathematics and Artificial Intelligence, 23:229-245, 1998.

[3] H. Fleischer, O. Kullmann, S, Szeider: Polynomial-time recognition of minimal unsatisfiable formulas with fixed clause-variable difference, Theoret. Comput. Sci., 289(1):503-516, 2002.

[4] S. Fortune, J. E. Hopcroft, J. Wyllie, The Directed Subgraph Homeomorphism Problem. Theoretical Computer Science, 10(2):111-121, 1980.

[5] K. Iwama and E. Miyano, Intractability of read-once resolution, Proceedings Structure in Complexity Theory, 10th Annual Conference (IEEE), 2936, 1995. 
[6] H. Kleine Büning, X. Zhao, The Complexity of Read-Once Resolution, Annals of Mathematics and Artificial Intelligence, 36(4):419-435, 2002.

[7] H. Kleine Büning, X. Zhao, On the structure of some classes of minimal unsatisfiable formulas. Discrete Applied Mathematics, 130:185207, 2003.

[8] C. H. Papadimitriou and D. Wolfe. The complexity of facets resolved. J. of Computer and System Sciences, 37:2-13, 1988.

[9] S. Even, A. Itai, A. Shamir, On the Complexity of Timetable and Multicommodity Flow Problems, SIAM Journal on Computing, 5:691-703, 1976.

[10] P. Beame and T. Pitassi, Simplified and improved resolution lower bounds, 37th Annual Symposium on Foundations of Computer Science, IEEE, 274-282, 1996.

[11] P. Buss and T. Pitassi, Resolution and the weak pigeonhole principle, CSL: 11th Workshop on Computer Science Logic. LNCS, Springer-Verlag, 1997.

[12] P. Beame and T. Pitassi, Propositional proof complexity: Past, present, future, Bulletin of the EATCS, 65:66-89, 1998.

[13] S. Cook and R. Reckhow, Time bounded random access machines, Journal of Computer and System Sciences, 7(4):354-375, 1973.

[14] A. Haken, The intractability of resolution, Theoretical Computer Science, 39(2-3):297-308, 1985.

[15] J. Robinson, A machine-oriented logic based on the resolution principle, J. ACM, 12(1):23-41, 1965.

[16] A. Robinson and A. Voronkov, editors, Handbook of Automated Reasoning.

[17] S. Szeider, NP-Completeness of Refutability by Literal-Once Resolution. Automated Reasoning: First International Joint Conference, 168-181, 2001.

[18] A. Urquhart, The complexity of propositional proofs, The Bulletin of Symbolic Logic, 1(4):425-467, 1995. 


\section{A ROR Refutation Example}

We now apply read-once resolution refutation to generate a refutation of the 2SAT instance specified by Formula (1).

$$
\begin{array}{rll}
\left(x_{1}, x_{2}\right) & \left(\neg x_{1}, x_{3}\right) & \left(\neg x_{1}, x_{4}\right) \\
\left(\neg x_{2}, x_{3}\right) & \left(\neg x_{2}, x_{4}\right) & \left(\neg x_{3}, x_{5}\right) \\
\left(\neg x_{3}, x_{6}\right) & \left(\neg x_{4}, \neg x_{5}\right) & \left(\neg x_{4}, \neg x_{6}\right)
\end{array}
$$

The application of this read-once resolution refutation to Formula (1) can be seen in Figure 2.

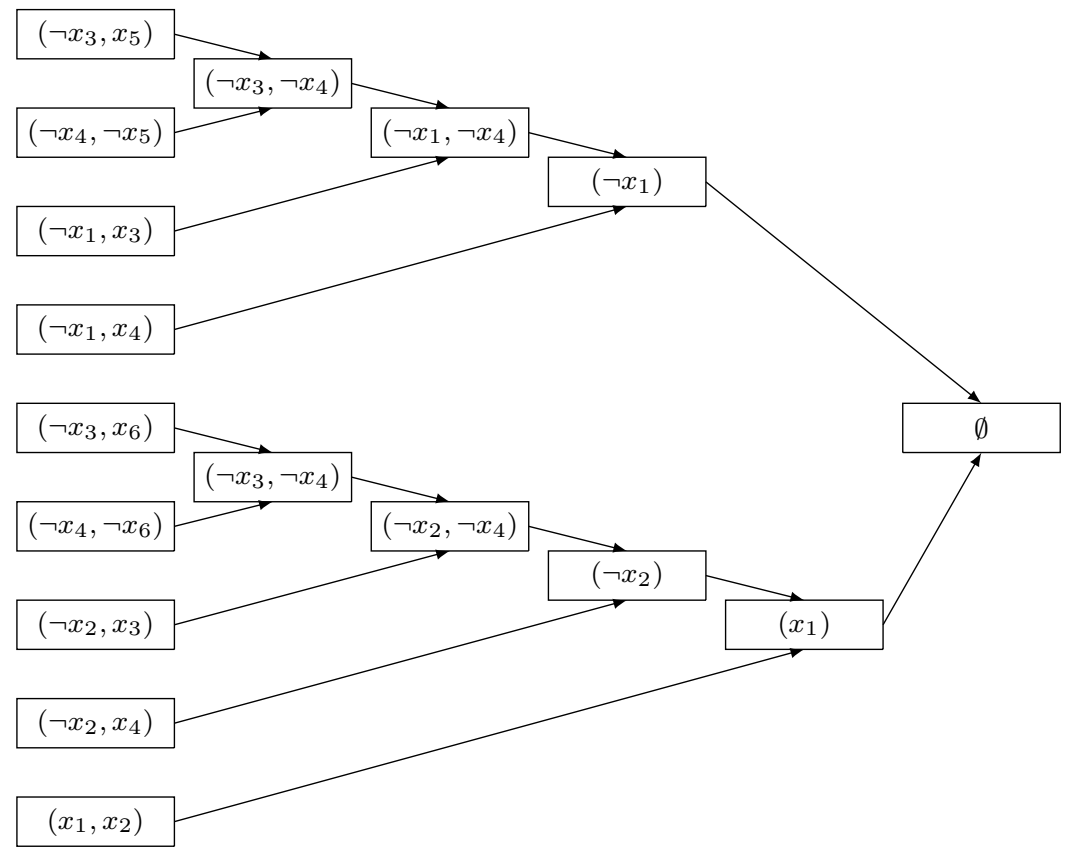

Figure 2: Read-Once Refutation

Note that the clause $\left(\neg x_{3}, \neg x_{4}\right)$ is used twice. However, this is still a read-once refutation since each time the clause $\left(\neg x_{3}, \neg x_{4}\right)$ is derived different clauses from the original formula are used.

\section{B Non MU(1) ROR refutation}

Example (3): Let

$$
\begin{aligned}
\Phi= & \{(\neg x \rightarrow a),(a \rightarrow b),(a \rightarrow c),(c \rightarrow b), \\
& (b \rightarrow x),(b \rightarrow \neg x),(x \rightarrow a)\} .
\end{aligned}
$$

$\Phi$ is not minimal unsatisfiable and has deficiency 2. Minimal unsatisfiable sub-formulas of $\Phi$ are:

$$
\begin{aligned}
\Phi_{1}= & \{(\neg x \rightarrow a),(a \rightarrow b),(b \rightarrow x),(b \rightarrow \neg x),(x \rightarrow a)\} \\
\Phi_{2}= & \{(\neg x \rightarrow a),(a \rightarrow c),(c \rightarrow b), \\
& (b \rightarrow x),(b \rightarrow \neg x),(x \rightarrow a)\} .
\end{aligned}
$$

Note that $\Phi_{1}$ and $\Phi_{2}$ have deficiency 1 . There is no read-once resolution refutation for $\Phi_{1}$ or $\Phi_{2}$. However, $\Phi$ has the following read-once resolution refutation: 
1. $(\neg x \rightarrow a) \wedge(a \rightarrow c) \frac{1}{R E S}(\neg x \rightarrow c)$.

2. $(\neg x \rightarrow c) \wedge(c \rightarrow b) \mid \frac{1}{R E S}(\neg x \rightarrow b)$.

3. $(\neg x \rightarrow b) \wedge(b \rightarrow x) \frac{1}{\operatorname{RES}}(x)$.

4. $(x \rightarrow a) \wedge(a \rightarrow b) \frac{1}{R E S}(x \rightarrow b)$.

5. $(x \rightarrow b) \wedge(b \rightarrow \neg x) \frac{1}{R E S}(\neg x)$.

6. $(\neg x) \wedge(x) \frac{1}{R E S} \sqcup$.

From $\Phi$ we can construct the graph $G$ as follows:

1. For each literal in $\Phi$, create a vertex in $G$.

2. For each implication $(x \rightarrow a)$ in $\Phi$, create the edge $(x \rightarrow a)$ in $G$.

The result of this can be seen in Figure 3.

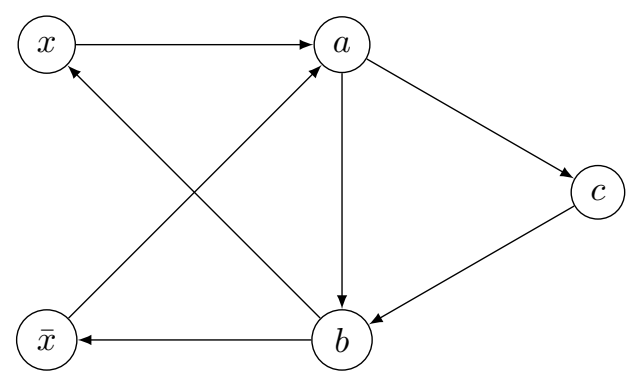

Figure 3: Edge-Disjoint Cycle: $x$ to $\bar{x}$ and $\bar{x}$ to $x$

The paths $\neg x, a, b, x$ and $x, a, c, b, \neg x$ form an edge-disjoint cycle from $\neg x$ to $x$ and from $x$ to $\neg x$. These paths correspond to the follow sub-formulas of $\Phi$ :

$$
\begin{aligned}
F_{x}^{x} & =(\neg x \rightarrow a) \wedge(b \rightarrow x) \wedge(a \rightarrow b) \\
F_{\neg x}^{\neg x} & =(a \rightarrow c) \wedge(c \rightarrow b) \wedge(x \rightarrow a) \wedge(b \rightarrow \neg x) .
\end{aligned}
$$

The following summarizes our observations:

1. The formula $\Phi$ is in ROR, but not minimal unsatisfiable. Moreover, any minimal unsatisfiable sub-formula of $\Phi$ has no read-once resolution refutation.

2. The formula $\Phi$ is minimally ROR and $\Phi$ has deficiency greater than 1 .

3. The formula $\Phi$ has two sub-formulas formulas in $\mathrm{MU}(1)$.

4. The formula $\Phi$, when considered as directed graph, has an edge-disjoint cycle from $\neg x$ to $x$ and from $x$ to $\neg x$. Each path in this cycle corresponds to a sub-formula of $\Phi$. 